ESAIM: PROCEEDINGS, January 2014, Vol. 44, p. 140-149

SMAI Groupe MAS - Journées MAS 2012 - Prix Neveu

\title{
WALKS IN THE QUARTER PLANE: ANALYTIC APPROACH AND APPLICATIONS
}

\author{
KILIAN RASCHEL ${ }^{1}$
}

\begin{abstract}
In this survey we present an analytic approach to solve problems concerning (deterministic or random) walks in the quarter plane. We illustrate the recent breakthroughs in that domain with two examples. The first one is about the combinatorics of walks confined to the quarter plane, and more precisely about the numbers of walks evolving in the quarter plane and having given length, starting and ending points. We show how to obtain exact and asymptotic expressions for these numbers, and how to find the algebraic nature of their generating function. The second example deals with population biology, and more specifically with the extinction probabilities of certain flower populations.
\end{abstract}

\section{INTRODUCTION}

\subsection{Motivations and organization of the paper}

For more than one decade, walks confined to the positive quarter plane (or positive quadrant) $\mathbf{Z}_{+}^{2}$ attract much attention from the mathematical community. The main motivation to study these walks lies in the number and in the richness of applications. Indeed, walks in the quarter plane are related to

- combinatorics, as many combinatorial objects (like maps, permutations, trees, Young tableaux) can be encoded by lattice walks, in particular by walks in the quarter plane, see [1,2];

- complex analysis, as it turns out that the inclusion $\mathbf{Z}_{+}^{2} \subset \mathbf{C}$ is convenient for applying methods from complex analysis, see $[6-8,11,13,15]$;

- finance, as the dynamics of certain limit order books may be approximated by random processes in the quarter plane, see [5];

- population biology, as the quarter plane is the natural space to parametrize any two-dimensional population, see [12] for an example;

- probability theory, since random walks in cones (e.g., quantum random walks, non-colliding random walks) are a very actual topic;

- queueing theory, as any two-dimensional queue (and many of them are important for the theory, see [4]) can be modeled by random walks in the quarter plane;

- etc.

The present article aims at summarizing part of our contributions to that domain obtained in the $\mathrm{PhD}$ thesis [15] and afterwards $[8,11,12]$. We choose to focus here on two particular applications, that we consider representative of the variety of the problems related to (deterministic or random) walks in the quarter plane. The first topic we shall tackle is an instance of a most classical problem in combinatorics, which consists in counting walks

${ }^{1}$ CNRS \& Université de Tours, Laboratoire de Mathématiques et Physique Théorique (UMR 7350) \& Fédération Denis Poisson (FR 2964). Email: Kilian.Raschel@lmpt.univ-tours.fr

(C) EDP Sciences, SMAI 2013 

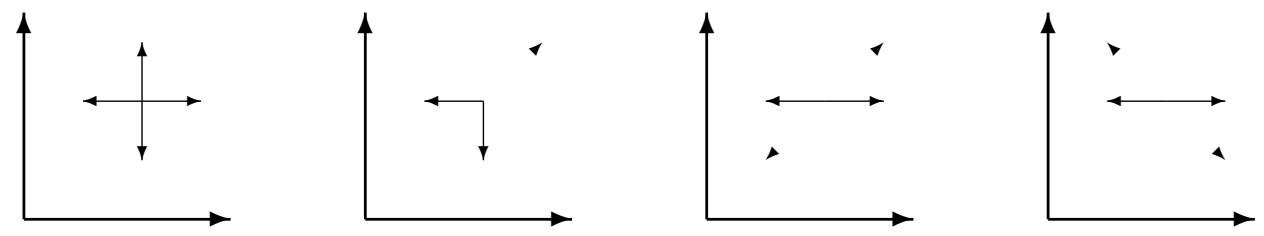

Figure 1. Four examples, known as the simple, Kreweras', Gessel's and Gouyou-Beauchamps' walks

confined to some domains (here, the quarter plane). We shall then move to an application in biology, dealing with the extinction probability of certain flower populations. This survey is organized as follows: We shall first briefly present these two applications, in Sections 1.2 and 1.3. Next, in Section 1.4, we will sketch the main ideas of the analytic methods that we used in $[8,11,12,15]$ to study these problems. Then, in the main Sections 2 and 3 , we will return to the two applications, in more details; in particular, there we shall present our contributions.

\subsection{Counting walks confined to the quarter plane}

A point in the lattice $\mathbf{Z}_{+}^{2}$ has eight neighbors, diagonal neighbors included. Choosing a subset $\mathcal{S}$ of the set of neighbors and considering it as the set of admissible steps of a walk in the lattice, we obtain one of $2^{8}=256$ possible models of walks in $\mathbf{Z}_{+}^{2}$ (see Figure 1 for some examples). Bousquet-Mélou and Mishna [2] showed that, after eliminating trivial cases, and also those which can be reduced to the walks in a half plane, there remain 79 inherently different models. The main questions of interest concern

$q_{\mathcal{S}}(i, j ; n)=\mid$ paths with jumps in $\mathcal{S}$, starting at $(0,0)$, ending at $(i, j)$, having length $n$ and staying in $\left.\mathbf{Z}_{+}^{2}\right\} \mid$.

They are the following:

(i) What is the expression of $q_{\mathcal{S}}(i, j ; n)^{1}$ ?

(ii) What is the asymptotic behavior of the number of walks ending at some given point or domain (for instance one axis), as their length goes to infinity?

(iii) What is the nature of the generating function of the numbers of walks: Is it holonomic ${ }^{2}$, and, in that case, algebraic or even rational ${ }^{3}$ ?

These (qualitative and quantitative) questions illustrate the variety of problems we address here. In Section 2 we shall present our contributions to problems (i) and (iii). For brevity, and also because the topic is a burning issue (and thus evolves very quickly), we choose here not to present results regarding (ii).

\subsection{Population biology}

A crucial motivation to study random walks in the quarter plane is that $\mathbf{Z}_{+}^{2}$ is the natural space to parametrize any two-dimensional population. In this survey we shall only consider one instance (taken from [12]) of such a two-dimensional population, but it is worth keeping in mind that many others could be similarly analyzed. The example we take here is the following: We consider a flower population of two types, say $a$ and $A$, for which we

\footnotetext{
${ }^{1}$ Of course, it is possible to count the numbers of trajectories; here, we ask ourselves if it is possible to find closed-form expressions for these numbers, or for their generating function.

${ }^{2}$ A function of one or several complex variables is said to be holonomic (or D-finite) if the vector space over the field of rational functions spanned by the set of all derivatives is finite dimensional. Any algebraic function is holonomic. In the case of one variable, this is tantamount to saying that the function is solution of a linear differential equation where the coefficients are rational functions (see [10, Appendix B]).

${ }^{3}$ Prima facie, question (iii) may appear less natural than (i) and (ii). Actually, it is motivated by (at least) two facts: First, it allows to classify the 79 models according to the nature of the generating function; second, if the counting function happens to be holonomic, then the answer to (ii) will be simpler to obtain, as the asymptotic behavior of coefficients of holonomic functions is well understood, see [10].
} 


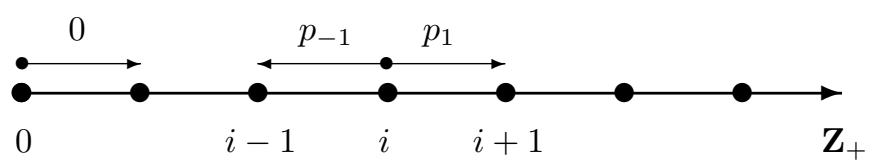

FIGURE 2. Transition probabilities for the gambler ruin problem

suppose that the reproduction if sporophytic (two individuals can have an offspring only if they have different types). A consequence of this hypothesis is that if it remains individuals of only one type, the population gets extinct. The interesting quantities are then these extinction probabilities. Further, the biological model (see Section 3 for more details) implies that the process $\left(X_{n}^{a}, X_{n}^{A}\right)_{n \geqslant 0}$ of the numbers of individuals having type $a$ and $A$, at the $n$-th generation, is a inhomogeneous random walk within the quarter plane, absorbed at the boundary. Accordingly, the generating function of the extinction probabilities satisfies a certain partial differential equation (PDE). In Section 3 we shall present the model in more details, as well as our methods and results concerning this PDE, and, accordingly, the extinction probabilities.

\subsection{Analytic approach}

Though the themes of the problems presented in Sections 1.2 and 1.3 are quite different, they have in common that they concern (random or deterministic) walks in the quarter plane $\mathbf{Z}_{+}^{2}$, and they can thus be analyzed via the same analytic methods, that we now briefly present.

The pioneered approach to solve problems dealing with walks in $\mathbf{Z}_{+}^{2}$ via the use of functional equations and complex analysis was proposed by Malyshev [13] in the 70s. At this time the main motivation was to study stationary probabilities for ergodic random walks, homogeneous inside of $\mathbf{Z}_{+}^{2}$ and reflected on the boundary. In [13], Malyshev obtained an expression for their generating function, in terms of infinite sums and products defined on a certain Riemann surface. Not a long time after, Fayolle and Iasnogorodski [6] initiated an approach to solve similar problems, that is based on boundary value problem theory. More recently [7], the three abovecited authors generalized and proposed new ways for this analytic approach.

Before describing this method, let us be interested in its analogue in dimension 1, through the example of the gambler ruin. Let us recall that the gambler ruin is a one-dimensional random process, where a gambler with finite wealth plays a (fair or not fair) game against an opponent with infinite wealth. Denote by $X(k)$ the wealth of the gambler at time $k \geqslant 0$. Then $(X(k))_{k \geqslant 0}$ is a random walk on $\mathbf{Z}_{+}$to the nearest neighbors (at each unit of time, the gambler wins or looses one unit of money), with probabilities $p_{1}$ to win and $p_{-1}$ to loose, starting from some initial state $i_{0}$ (the initial wealth), and absorbed at 0 (the gambler stops playing after his/her ruin); see Figure 2.

The quantity of greatest interest ${ }^{4}$ is the ruin probability $q_{i_{0}}=\mathbf{P}_{i_{0}}[\inf \{\ell \geqslant 1: X(\ell)=0\}<\infty]$. Other interesting quantities are the Green functions $\sum_{k \geqslant 1} \mathbf{P}_{i_{0}}[X(k)=i]$ (how many time, during his/her play, will the gambler have the wealth $i$ ?), or equivalently their generating function $Q_{i_{0}}(x)=\sum_{i \geqslant 1, k \geqslant 0} \mathbf{P}_{i_{0}}[X(k)=i] x^{i-1}$. A classical method (see [9, Chapter XIV, Sections 1-5]) to find all these quantities can be summarized as follows:

Step 1: Find a functional equation between the quantities of interest. In our case, we have:

$$
x\left[p_{1} x+p_{-1} x^{-1}-1\right] Q_{i_{0}}(x)=q_{i_{0}}-x^{i_{0}} .
$$

Indeed, it follows from our model that for $k \geqslant 0$,

$$
\mathbf{P}[X(k+1)=i]= \begin{cases}\mathbf{P}[X(k)=i-1] p_{1}+\mathbf{P}[X(k)=i+1] p_{-1} & \text { if } i \geqslant 2 \\ \mathbf{P}[X(k)=2] p_{-1} & \text { if } i=1 \\ \mathbf{P}[X(k)=0]+\mathbf{P}[X(k)=1] p_{-1} & \text { if } i=0\end{cases}
$$

\footnotetext{
${ }^{4}$ For us - but for the gamber too.
} 
Multiplying the latter by $x^{i}$ and summing with respect to $i \geqslant 1$ and $k \geqslant 0$ yields (1).

Step 2: Evaluate the functional equation at a well-chosen value of $x$, and find the unknown present in the right-hand side of the equation. In our case, the two solutions to $p_{1} x+p_{-1} x^{-1}-1=0$ are positive, and we denote by $\widetilde{x}$ the smallest one. Evaluating (1) at $\widetilde{x}$, the left-hand side vanishes, and we find $q_{i_{0}}=\widetilde{x}^{i_{0}}$.

Step 3: Come back to the functional equation, and obtain an expression for the unknown in the left-hand side of the equation. In our case, using (1) with the value of $q_{i_{0}}$ found in the second step, we obtain an expression for $Q_{i_{0}}(x)$, namely, $Q_{i_{0}}(x)=\left(q_{i_{0}}-x^{i_{0}}\right) /\left(x\left[p_{1} x+p_{-1} x^{-1}-1\right]\right)$.

Remark. After these three steps, we have found an expression for all the quantities of interest; this corresponds to problem (i) of Section 1.2. We would like to mention that this three-step approach is actually more powerful. Indeed, we can also deduce the algebraic nature (problem (iii)) of the function $Q_{i_{0}}(x)$ : It is a rational function. We can finally find the asymptotics of its coefficients (problem (ii)), using its behavior near its dominant singularity $\widetilde{x}$ and classical singularity analysis [10, Chapter VI].

To conclude Section 1.4, we return to our initial problem (the quarter plane), and we consider the extension of this three-step approach to the dimension 2:

Step 1. Typically $[12,15]$, for walks in the quarter plane, the functional equation takes the form

$$
K(x, y) Q(x, y)=a(x, y) q(x)+b(x, y) \widetilde{q}(y)+c(x, y) q_{0}+d(x, y)
$$

where $K, a, b, c, d$ are known functions, while $Q, q, \widetilde{q}, q_{0}$ are not. Indeed, as examples, see (4) for the counting problem of Section 1.2, and (11) for the population biology problem of Section 1.3. The first step is in some sense always simple, as the functional equation (if any!) is intrinsic to the problem, and can be easily derived.

Step 2. The analogue of the second step, for the dimension 2, would be to evaluate the functional equation (2) at some $(x, y)$ such that $K(x, y)=0$. Doing this, we do have an equation between $q, \widetilde{q}, q_{0}$, but this is not enough to obtain expressions for the latter functions ${ }^{5}$. In fact, the inventiveness of Fayolle, Iasnogorodski and Malyshev consisted in finding another way for this second step. Roughly said, their idea was the following: Evaluate (2) at infinitely many and well-chosen $(x, y)$ such that the kernel $K(x, y)=0$. This way, we obtain

infinitely many equations between the unknowns $q, \widetilde{q}, q_{0}$. By a suitable reformulation of all these equations, we can show that $q, \widetilde{q}$ satisfy certain boundary value problems, and eventually obtain expressions for that functions. The quantity $q_{0}$ then typically follows by a normalizing argument. We refer to $[6,7,12,13,15]$ for more details.

Step 3. As for the dimension 1 case, the third step is easy: It suffices to use the functional equation (2).

\section{Counting Walks CONFIned to The QuARTER Plane}

This part is devoted to the counting of walks in the quarter plane. We first (Section 2.1) present the context; then (Section 2.2) we state the significant results obtained in $[8,11,15]$.

\subsection{General context}

Going back to the 79 models of walks presented in Section 1.2 (see Figure 1 for four well-known models), the central object for their study is the following: Denoting, as in Section 1.2 , by $q_{\mathcal{S}}(i, j ; n)$ the number of paths confined to $\mathbf{Z}_{+}^{2}$, with jumps in a given step set $\mathcal{S} \subset\{0, \pm 1\}^{2}$, having length $n$, starting at $(0,0)$ and ending at $(i, j)$, their generating function is defined as

$$
Q(x, y ; z)=Q_{\mathcal{S}}(x, y ; z)=\sum_{i, j, n \geqslant 0} q_{\mathcal{S}}(i, j ; n) x^{i} y^{j} z^{n}
$$

\footnotetext{
${ }^{5}$ It was enough to do so for the dimension 1 , since there was only one unknown function in the right-hand side of the functional equation, and since in addition this unknown function was just a real number.
} 

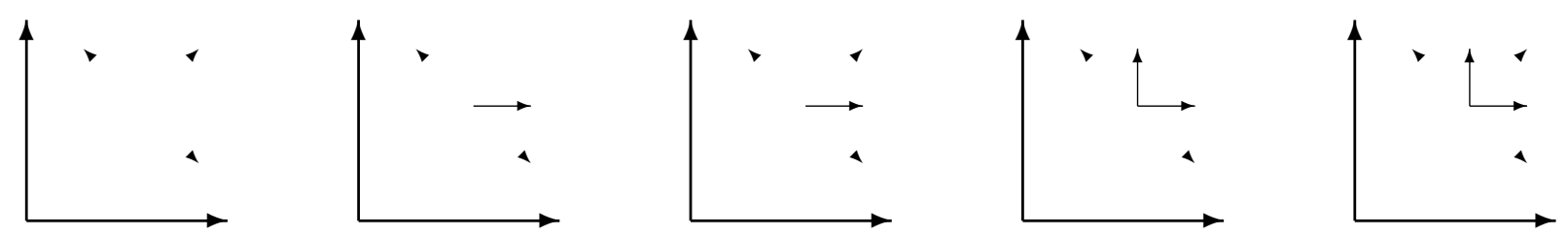

Figure 3. The 5 singular walks in the classification of [2]

A fundamental observation is that it satisfies a certain functional equation. Precisely, introducing the kernel of the step set $K(x, y ; z)=K_{\mathcal{S}}(x, y ; z)=x y z\left(\sum_{(i, j) \in \mathcal{S}} x^{i} y^{j}-1 / z\right)$, we have

$$
K(x, y ; z) Q(x, y ; z)=K(x, 0 ; z) Q(x, 0 ; z)+K(0, y ; z) Q(0, y ; z)-K(0,0 ; z) Q(0,0 ; z)-x y .
$$

This functional equation is the fundamental starting point of our study - and is also so for almost every other works on the topic. It relates the trivariate generating function $Q(x, y ; z)$ to the bi- and univariate generating functions $Q(x, 0 ; z), Q(0, y ; z)$ and $Q(0,0 ; z)$, which count the walks which end on the borders. Notice that (4) simply follows from the step by step construction of the walks; its proof may be found in [2, Section 4].

In this way, for the purpose of answering questions (i), (ii), and (iii) stated at the beginning of this paper, it suffices to solve (4). A key idea then is to consider a certain group, introduced in [13] in a probabilistic context, and called the group of the walk. This is a group of birational transformations of $\mathbf{C}^{2}$ which leave invariant the step generating function $\sum_{(i, j) \in \mathcal{S}} x^{i} y^{j}$. Precisely, this is the group $W=W_{\mathcal{S}}=\langle\Psi, \Phi\rangle$ generated by

$$
\Psi(x, y)=\left(x, \frac{\sum_{(i,-1) \in \mathcal{S}} x^{i}}{\sum_{(i,+1) \in \mathcal{S}} x^{i}} \frac{1}{y}\right), \quad \Phi(x, y)=\left(\frac{\sum_{(-1, j) \in \mathcal{S}} y^{j}}{\sum_{(+1, j) \in \mathcal{S}} y^{j}} \frac{1}{x}, y\right) .
$$

Obviously $\Psi \circ \Psi=\Phi \circ \Phi=\mathrm{id}$, and $W$ is a dihedral group, thus of order even (possibly infinite) and at least four. This order is calculated in [2] for each of the 79 cases: 23 walks admit a finite group (of order four, six or eight), and the 56 others have an infinite group. For instance, for the simple walk (on the left on Figure 1), $\Psi(x, y)=(x, 1 / y)$ and $\Phi(x, y)=(1 / x, y)$, so that $W$ is composed of the four following elements: The identity, $\Psi(x, y), \Phi(x, y)$, and $\Psi \circ \Phi(x, y)=\Phi \circ \Psi(x, y)=(1 / x, 1 / y)$. Likewise, the interested reader can check that the group of Kreweras', Gessel's and Gouyou-Beauchamps' walks has order six, eight and eight, respectively.

For the 23 walks with a finite group, the answers to questions (i) and (iii) have been given recently. Indeed, the article [2] successfully treats 22 of the 23 models associated with a finite group: The series (3) is made explicit and is shown to be either algebraic or transcendental but holonomic. As for the 23rd walk (namely, Gessel's walk represented in Figure 1), Bostan and Kauers [1] have given a computer-aided proof of the algebraicity of the function (3).

Concerning the 56 walks with an infinite group, the 5 represented on Figure 3 are special; they are called singular. Of these, 2 cases are solved: In [14], Mishna and Rechnitzer have considered the two walks on the left on Figure 3, have made explicit the series (3), and have shown that it is non-holonomic. Thus, before [15], there remained 54 walks whose status was unsettled, as regards (i), (ii), and (iii).

\subsection{Contributions of $[8,11,15]$ to the combinatorics of walks in the quarter plane}

Before stating our contributions to (i) and (iii) (we recall that for brevity, and also because the subject is quite topical, we shall not mention here results around (ii)), it is important to note that we needed some changes in the analytic approach: Indeed, our functional equation (4) is different from the generic functional equation (2), as it involves an additional variable $z$. Let us illustrate how to deal with this new variable $z$ for the gambler ruin problem. The ruin probability $q_{i_{0}}$ should be replaced by $q_{i_{0}}(z)=\sum_{k \geqslant 0} \mathbf{P}_{i_{0}}[\inf \{\ell \geqslant 1: X(\ell)=0\}=k] z^{k}$, and 
the generating function $Q_{i_{0}}(x)$ of the Green functions by $Q_{i_{0}}(x ; z)=\sum_{i \geqslant 1, k \geqslant 0} \mathbf{P}_{i_{0}}[X(k)=i] x^{i-1} z^{k}$. Equation (1) becomes (its proof is similar to that of (1), so we omit it):

$$
x\left[z\left(p_{1} x+p_{-1} x^{-1}\right)-1\right] Q_{i_{0}}(x ; z)=q_{i_{0}}(z)-x^{i_{0}} .
$$

This example shows that adding the variable $z$ in the functional equation eventually provides more accurate information: We are now able not only to compute the ruin probability $q_{i_{0}}=q_{i_{0}}(1)$, but also the ruin probability at any time $k, \mathbf{P}_{i_{0}}[\inf \{\ell \geqslant 1: X(\ell)=0\}=k]$. To that aim we proceed as in Section 1.4: Let $x(z)$ be the unique power series solution to $z\left(p_{1} x(z)+p_{-1} x(z)^{-1}\right)-1=0$. Evaluating Equation (6) at $x(z)$, we find $q_{i_{0}}(z)=x(z)^{i_{0}}$; the ruin probabilities at fixed time are the Taylor coefficients of the latter power series.

\section{Explicit expression for the generating function (question (i))}

In our first work [15, Chapter A] (in collaboration with Irina Kurkova) concerning combinatorics, we found an expression for $Q(x, y ; z)$ in the case of Gessel's walk. The formula we obtained can be written as a sum of three integrals over segments

$$
\int f(t ; z) \frac{\partial_{t} w(t ; z)}{w(t ; z)-w(u ; z)} \mathrm{d} t, \quad u=x, y, 0
$$

where $f$ is an algebraic function and $w$ a certain conformal mapping, algebraic in the case of Gessel's walk. The main motivation to consider first Gessel's walk was that the latter was quite à la mode [1,2] in the combinatorics community. Still today, there remain interesting open problems around Gessel's model, as to show that the expression (7) we have obtained for $Q(x, y ; z)$ is an algebraic function (indeed, there does not exist a computerfree proof of this fact).

In [15, Chapter B], we then considered all 79 models, and we found an expression for $Q(x, y ; z)$ in a unified way. In all cases, $Q(x, y ; z)$ can be expressed as a sum of three integrals $(7)$ along segments. The main difference between all 79 models lies in the conformal mapping $w$ inside of these integrals.

The existence of function $w$ was easy to show, but finding an expression for it turned out to be a challenging problem (as often in the theory of conformal mapping), and this actually was our main contribution. We found explicitly this conformal mapping for all models, and we emphasized the following link between its algebraic nature, the finiteness of the group $W$ and the sign of the covariance $\sum_{(i, j) \in \mathcal{S}} i j-\left(\sum_{(i, j) \in \mathcal{S}} i\right)\left(\sum_{(i, j) \in \mathcal{S}} j\right)$ of the step set $\mathcal{S}$ : The function $w$ is rational if the group is finite and the covariance is negative or zero; it is algebraic if the group is finite and the covariance is positive; it is non-holonomic if the group is infinite. In the last case, we proved that $w$ admits the expression $w(t ; z)=\wp\left(g\left(\widetilde{\wp}^{-1}(t) ; z\right)\right)$, where $g$ is an affine function, and where $\wp, \widetilde{\wp}$ are Weierstrass elliptic functions associated with different lattices, that in addition depend on $z$.

To summarize, [15, Chapters A and B] proposed a unified answer to problem (i). For 54 out of the 79 models, this answer was given for the first time.

\section{Algebraic nature of the generating function (question (iii))}

Although we had an explicit integral expression for $Q(x, y ; z)$ and we knew the nature of the functions present in the integrals, it remained an open problem to deduce the algebraic nature of $Q(x, y ; z)$. This is why we had to find alternative ideas for solving (iii).

The first partial answer we obtained in this direction is the topic of [8] (coauthored with Guy Fayolle). There we (re)found, after [1,2], the nature of the function $Q(x, y ; z)$ for any 23 models with finite group, using methods that are completely different from that used in $[1,2]$. Indeed we used an algebraic approach, based on Galois theory. A worthwhile consequence of our work was that it was the first time that a computer-free proof of the algebraicity of the (bivariate) Gessel's generating function was obtained. However, this approach is intrinsic to the finite group case.

The papers $[1,2,8,15]$ showed a posteriori that for the 23 models with a finite group, the nature of $Q(x, y ; z)$ is related to that of the conformal mapping $w$ (they both are holonomic). This suggests that this link also exists 

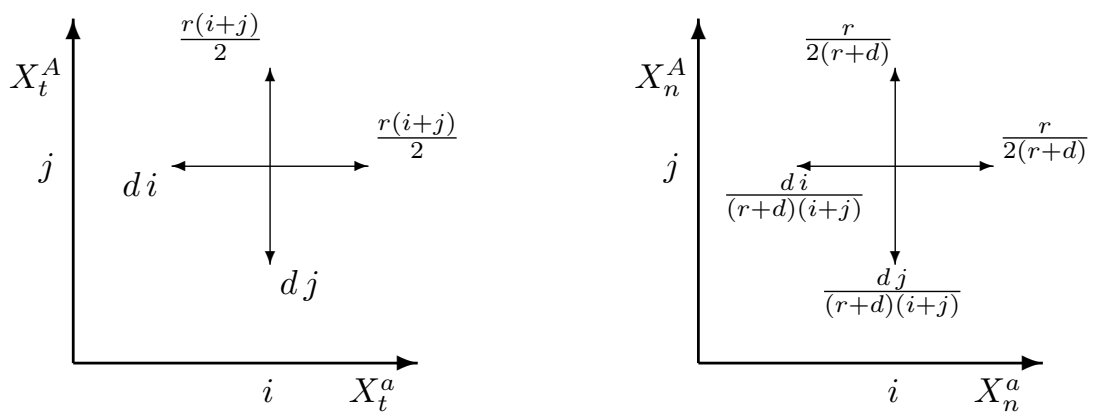

Figure 4. Transition rates (for the continuous-time model $\left.\left(X_{t}^{a}, X_{t}^{A}\right)_{t \in \mathbf{R}_{+}}\right)$and transition probabilities (for the discrete-time model $\left.\left(X_{n}^{a}, X_{n}^{A}\right)_{n \geqslant 0}\right)$. The parameters $r$ and $d$ are the rates of production and of lifetime of the individuals, respectively; see below for more details.

for walks with infinite group, and the conjecture (stated in [2]) was that $Q(x, y ; z)$ is then non-holonomic. This conjecture has been proved only very recently, in [11]. The tools used there are analytic (Riemann surfaces, universal cover, branches of meromorphic functions, etc.), and we shall not present them in more details here. น

\section{Population Biology}

Our choice for the example of Section 3 is motivated by two main reasons: First, we would like to illustrate that many scientific domains (here, biology) lead to considering walks in the quarter plane. Second, after the generalization of the analytic approach of Section 2, that consisted in adding a third variable $z$, we want to introduce another very concrete extension of the analytic approach, dealing with inhomogeneities. The gambler ruin problem and the counting of walks already presented inhomogeneities, as the behavior was different depending on whether the walk evolved inside of the cone (the half line and the quarter plane) or on the boundary. In that section we shall consider quite different and more complex inhomogeneities, since we shall allow the behavior of the walk inside of the quarter plane to be dependent of the current position. For the gambler ruin of Section 1.4, this would mean allowing the transitions $p_{1}$ and $p_{-1}$ (see Figure 2) to depend on $i$. Techniques and results in the literature $[2,6,7,13]$ for random walks on positive quadrants mostly focus on the homogeneous case, and our opinion is that being able to obtain precise results (say, with the same level of precision as in Section 2) for such inhomogeneous walks inside of the quarter plane would have important consequences in many domains.

\subsection{General context}

We consider here the model of distyle plants with sporophytic reproduction and absence of pollen limitation introduced in Billiard and Tran [3]. As we shall see, this model will eventually lead to study the process on Figure 4 (see on the left for the related continuous-time model, and on the right for the associated discrete-time pure-jump process). Indeed, the main assumptions that we do here are the following:

- Each plant is diploid and is characterized by the two alleles that it carries at a locus.

- Only two alleles are available, say $a$ or $A$, and $A$ is dominant over $a$. Thus, the plants have genotypes $a a, a A$ or $A A$. The phenotype, i.e., the type of proteins carried by the pollen and stigmates, is then $a$, $A$ and $A$, respectively.

- Further, the reproduction is supposed to be (sexual and) self-incompatible. The latter prevents fecundation of the plant's ovule by its own pollen: Only pollen and stigmates with different proteins can give 
viable seeds, i.e., pollen of a plant of phenotype $A$ can only fecundate stigmates of a plant of phenotype $a$ and vice-versa.

Accordingly, seeds $A A$ cannot be created. We can therefore consider, without restriction, populations made of only individuals of genotypes $a a$ and $a A$. Each seed is then necessarily of genotype $a a$ or $a A$ with probability $1 / 2$. It is assumed that ovules are produced in continuous time at rate $r>0$ and that each ovule is fecundated to give a seed, provided there exists compatible pollen in the population. The lifetime of each individual follows an exponential distribution with mean $1 / d$, where $d>0$. Let us denote by $X_{t}^{a}$ and $X_{t}^{A}$ the number of individuals of genotype $a a$ (phenotype $a$ ) and $a A$ (phenotype $A$ ) at time $t \in \mathbf{R}_{+}$. The process $\left(X_{t}^{a}, X_{t}^{A}\right)_{t \in \mathbf{R}_{+}}$is a pure-jump Markov process with transitions represented on Figure 4.

When one of the phenotype $a$ or $A$ disappears, reproduction becomes impossible and the system gets extinct. This is this extinction phenomenon in which we are interested here. To that purpose, let us introduce the first time at which one of the two types gets extinct:

$$
\tau_{0}=\inf \left\{n \geqslant 0: X_{n}^{a}=0 \text { or } X_{n}^{A}=0\right\} .
$$

For $i, j \geqslant 0$, denote by

$$
a_{i, j}=\mathbf{P}_{(i, j)}\left[\tau_{0}<\infty\right], \quad P(x, y)=\sum_{i, j \geqslant 1} a_{i, j} x^{i} y^{j},
$$

the absorption probabilities and their generating function. By symmetry arguments, we have, for all $i, j \geqslant 0$, $a_{i, j}=a_{j, i}$. Moreover, for any $i, j \geqslant 0$ such that $i=0$ or $j=0$, we have $a_{i, j}=1$.

\subsection{Contributions of [12] to this population biology model}

In this part we present our contributions [12] to the population biology model introduced in Section 3.1. To this aim we consider the (now usual) decomposition into three main steps of the analytic approach, and for each step we shall explain the main differences that appear when analyzing this particular model.

Step 1. We first establish a functional equation for the generating function (9) of interest. To that purpose, we note that an easy consequence of the transition probabilities of our model (see Figure 4) is that the extinction probabilities satisfy the following equations, valid for any $i, j \geqslant 1$ :

$$
a_{i, j}=\frac{r}{2(r+d)} a_{i+1, j}+\frac{r}{2(r+d)} a_{i, j+1}+\frac{d j}{(r+d)(i+j)} a_{i, j-1}+\frac{d i}{(r+d)(i+j)} a_{i-1, j}
$$

We now multiply (10) by $x^{i} y^{j}$, and we sum with respect to $i, j \geqslant 1$. After reformulation (see [3, Section 3] for more details), we obtain that the generating function $P(x, y)$ satisfies

$$
A P(x, y)=h(x, y, P)
$$

where

$$
\begin{aligned}
A P(x, y) & =Q(x, y) \frac{\partial P}{\partial x}(x, y)+Q(y, x) \frac{\partial P}{\partial y}(x, y)+R(x, y) P(x, y), \\
Q(x, y) & =(r+d) x-\frac{r}{2}-\frac{r}{2} \frac{x}{y}-d x^{2}, \\
R(x, y) & =\frac{r}{2 x}+\frac{r}{2 y}-d x-d y, \\
h(x, y, P) & =-\frac{r}{2}\left(\sum_{i \geqslant 1} a_{i, 1} i x^{i}+\sum_{j \geqslant 1} a_{1, j} j y^{j}\right)+d\left(\sum_{i \geqslant 1} x^{i} y+\sum_{j \geqslant 1} y^{j} x\right) .
\end{aligned}
$$

The only (but crucial!) difference between the functional equation (11) and the generic equation (2) is that the left-hand side of (11) now involves partial derivatives of $P(x, y)$. 
Remark. At this stage, it is worth remarking that the order of the PDE (11) is strongly related to the particular form of the transition probabilities of the model we are considering. For instance, if instead of being linear in $i, j$, the transition rates on Figure 4 were of order two in $i, j$, the PDE would involve second-order derivatives of $P(x, y)$.

Incidentally, let us notice that another difficulty for solving (11) is that there is no more uniqueness of the solutions to (10): Indeed, the constant sequence equal to 1 is an obvious solution, but if $r>d$, we have $a_{i, j}<1$ for all $i, j \geqslant 1$ (see [3]); in other words, for any initial population $(i, j)$ with $i, j \geqslant 1$, there is no extinction of the population with positive probability.

Step 2. This is an open problem! We cannot use the previous idea anymore, namely, to evaluate the functional equation (11) at infinitely many points $(x, y)$ where the left-hand side of (11) vanishes. The reason of this is simply that for general values of the parameters $r$ and $d$, there are no points $(x, y)$ where simultaneously $R(x, y)=Q(x, y)=Q(y, x)=0$. We thus are interested in any ideas that the reader could have!

We now mention the following alternative possibilities for the (partial) computation of the unknowns (i.e., the absorption probabilities $\left.a_{i, 1}=a_{1, i}\right)$ :

- Of course, we can simulate the random walk we are considering, and, applying Monte-Carlo methods, obtain approximations (with confidence intervals) for the absorption probabilities, see [12, Section 4.1]. This method presents the advantage that it works not only for the $a_{i, 1}=a_{1, i}$, but also for any $a_{i, j}$.

- We can also use deterministic methods, which, starting from the recurrence relations (10), provide the $\left(a_{i, j}\right)_{0 \leqslant i, j \leqslant N}$ in terms of the $\left(a_{N, j}\right)_{0 \leqslant j \leqslant N}$ and $\left(a_{i, N}\right)_{0 \leqslant i \leqslant N}$, see [12, Section 4.2]. A disadvantage of this approach is that we have to find the boundary values $\left(a_{N, j}\right)_{0 \leqslant j \leqslant N}$ and $\left(a_{i, N}\right)_{0 \leqslant i \leqslant N}$. On the bright side, the computational cost is good, when compared with the Montel-Carlo method.

- Finally, for large $i$ but bounded $j$ (or, symmetrically, large $j$ and bounded $i$ ), we can compute probabilities $a_{i, j}$ by making approximations with one-dimensional random walks, see [12, Section 2.2]. Indeed, if $i$ is large and $j$ is bounded, with a big probability, if the random walk eventually hits one axis, it will hit the vertical axis.

Step 3. For the functional equation (1) corresponding to the gambler ruin problem, for that (4) related to our combinatorial problem, or else for the typical functional equation (2), the third step was quite simple: It was an obvious consequence of the second step and of the functional equation. In the case of the equation (11), even if we suppose that we have found an expression for the right-hand side $h(x, y, P)$ (i.e., even if we solved the second step above), the third step becomes quite involved, as we have to solve the PDE (11). However, in our particular case, this happens to be doable, using tools from PDE theory (for details, see [12, Section 3]). 4

\section{ACKNOWLEDGments}

We wish to thank Olivier Durieu and an anonymous referee for useful comments on the present manuscript.

\section{REFERENCES}

[1] A. Bostan, and M. Kauers. The complete generating function for Gessel walks is algebraic. Proc. Amer. Math. Soc. 432 (2010) 3063-3078.

[2] M. Bousquet-Mélou, and M. Mishna. Walks with small steps in the quarter plane. Contemp. Math. 520 (2010) 1-40.

[3] S. Billiard, and V. C. Tran. A general stochastic model for sporophytic self-incompatibility. J. Math. Biol. 64 (2012) 163-210.

[4] J.W. Cohen, and O.J. Boxma. Boundary value problems in queueing system analysis. North-Holland Publishing Co., Amster$\operatorname{dam}(1983)$.

[5] R. Cont, and A. de Larrard. Price dynamics in a Markovian limit order book market. SIAM J. Financial Math. 4 (2013) 1-25.

[6] G. Fayolle, and R. Iasnogorodski. Two coupled processors: the reduction to a Riemann-Hilbert problem. Z. Wahrsch. Verw. Gebiete 47 (1979) 325-351.

[7] G. Fayolle, R. Iasnogorodski, and V. Malyshev. Random walks in the quarter plane. Springer-Verlag, Berlin (1999).

[8] G. Fayolle, and K. Raschel. On the holonomy or algebraicity of generating functions counting lattice walks in the quarter plane. Markov Process. Related Fields 16 (2010) 485-496.

[9] W. Feller. An introduction to probability theory and its applications. Vol. I. John Wiley and Sons, Inc., New York (1957). 
[10] P. Flajolet, and R. Sedgewick. Analytic combinatorics. Cambridge University Press, Cambridge (2009).

[11] I. Kurkova, and K. Raschel. On the functions counting walks with small steps in the quarter plane. Publ. Math. Inst. Hautes Études Sci. 116 (2012) 69-114.

[12] P. Lafitte-Godillon, K. Raschel, and V.C. Tran. Extinction probabilities for a distylous plan population modeled by an inhomogeneous random walk on the positive quadrant. SIAM J. Appl. Math. 73 (2013) 700-722.

[13] V. Malyshev. Positive random walks and Galois theory. Uspehi Mat. Nauk 26 (1971) 227-228.

[14] M. Mishna, and A. Rechnitzer. Two non-holonomic lattice walks in the quarter plane. Theor. Comput. Sci. 410 (2009) 36163630.

[15] K. Raschel. Chemins confinés dans un quadrant. Thèse de doctorat de l'Université Paris 6. (2010). 